\title{
Damage detection of curved member based on the changes in curvatures
}

\author{
Lin $\mathrm{Li}^{1, \mathrm{a},{ }^{*}}$ and $\mathrm{HaO} \mathrm{Xu}^{2, \mathrm{~b}}$ \\ ${ }^{1}$ Fujian Jiangxia University, Fuzhou, Fujian, P. R. China \\ ${ }^{2}$ Huazhong University of Science and Technology, Wuhan, Hubei, P.R. China \\ alilines@aliyun.com, b942261537@qq.com
}

Keywords: Damage detection, curved member, curvature.

\begin{abstract}
A damage location method based on the changes in curvatures was developed for curved structural member. First, the curves of intact and damage members were fitted using least square method. Then, the curvatures of intact and damage members were calculated by the fitted curves. Finally, the damage was located using the changes in the curvatures between intact and damage members. A numerical example of curved beam demonstrated the effectiveness of the developed method.
\end{abstract}

\section{Introduction}

The subway brings convenience to people's travel, however, tunnel defects is increasingly prominent [1]. The defects not only affect the use of the tunnel, but also increase the cost of maintaining the tunnel. They can even endanger traffic safety and affect the normal operation of trains [2]. Therefore, early detection of damage to the tunnel structure and timely maintenance are of great social and economic importance. Segments are the main bearing members of the shield tunnel. Unlike conventional structural members, the segments are curved members. Most damage detection methods are developed for straight members $[3,4]$. Therefore, it is of great significance to explore effective damage detection methods for curved members.

\section{Curve fitting based on least square method}

For a given set of data, $\left(x_{i}, y_{i}\right)(i=1,2, \cdots, N)$, the following function can be found

$$
f(x)=a_{0} f_{0}+a_{1} f_{1}+\ldots+a_{m} f_{m} .
$$

Which make the equation, $\sum_{i=1}^{N} \omega\left(x_{i}\right)\left[y_{i}-f\left(x_{i}\right)\right]^{2}$, minimum. Where $f_{0}, f_{1}, \ldots, f_{m}$ are a set of linearly independent functions, and $a_{0}, a_{1}, \ldots, a_{m}$ are corresponding undetermined coefficients. $\omega\left(x_{i}\right)$ is weight function, which reflects the degree of importance of the point $\left(x_{i}, y_{i}\right)$.

In order to make $\sum_{i=1}^{N} \omega\left(x_{i}\right)\left[y_{i}-f\left(x_{i}\right)\right]^{2}$ minimum, the following equations must be satisfied

$$
\left[\begin{array}{cccc}
\left(f_{0}, f_{0}\right) & \left(f_{0}, f_{1}\right) & \ldots & \left(f_{0}, f_{m}\right) \\
\left(f_{1}, f_{0}\right) & \left(f_{1}, f_{1}\right) & \ldots & \left(f_{1}, f_{m}\right) \\
\vdots & \vdots & \ddots & \vdots \\
\left(f_{m}, f_{0}\right) & \left(f_{m}, f_{1}\right) & \ldots & \left(f_{m}, f_{m}\right)
\end{array}\right]\left[\begin{array}{c}
a_{0} \\
a_{1} \\
\vdots \\
a_{m}
\end{array}\right]=\left[\begin{array}{c}
\left(f_{0}, Y\right) \\
\left(f_{1}, Y\right) \\
\vdots \\
\left(f_{m}, Y\right)
\end{array}\right] .
$$


Where $Y=\left(y_{1}, y_{2} \ldots, y_{n}\right)^{\mathrm{T}}$, and $\left(f_{s}, f_{t}\right)=\sum_{i=1}^{N} \omega\left(x_{i}\right) f_{s}\left(x_{i}\right) f_{t}\left(x_{i}\right)$. The undetermined coefficients of $a_{0}, a_{1}, \ldots, a_{m}$ can be solved from the Eq. (2).

Let $f_{0}=1, f_{1}=x, \ldots, f_{m}=x^{m}$, Eq. (2) has the following form

$$
\left[\begin{array}{cccc}
\sum_{i=1}^{N} \omega_{i} & \sum_{i=1}^{N} \omega_{i} x_{i} & \ldots & \sum_{i=1}^{N} \omega_{i} x_{i}^{m} \\
\sum_{i=1}^{N} \omega_{i} x_{i} & \sum_{i=1}^{N} \omega_{i} x_{i}^{2} & \ldots & \sum_{i=1}^{N} \omega_{i} x_{i}^{m+1} \\
\vdots & \vdots & \ddots & \vdots \\
\sum_{i=1}^{N} \omega_{i} x_{i}^{m} & \sum_{i=1}^{N} \omega_{i} x_{i}^{m+1} & \ldots & \sum_{i=1}^{N} \omega_{i} x_{i}^{2 m}
\end{array}\right]\left[\begin{array}{c}
a_{0} \\
a_{1} \\
\vdots \\
a_{m}
\end{array}\right]=\left[\begin{array}{c}
\sum_{i=1}^{N} \omega_{i} y_{i} \\
\sum_{i=1}^{N} \omega_{i} x_{i} y_{i} \\
\vdots \\
\sum_{i=1}^{N} \omega_{i} x_{i}^{m} y_{i}
\end{array}\right] .
$$

When $\omega_{i}=1$, Eq. (3) becomes

$$
\left[\begin{array}{cccc}
\sum_{i=1}^{N} 1 & \sum_{i=1}^{N} x_{i} & \ldots & \sum_{i=1}^{N} x_{i}^{m} \\
\sum_{i=1}^{N} x_{i} & \sum_{i=1}^{N} x_{i}^{2} & \ldots & \sum_{i=1}^{N} x_{i}^{m+1} \\
\vdots & \vdots & \ddots & \vdots \\
\sum_{i=1}^{N} x_{i}^{m} & \sum_{i=1}^{N} x_{i}^{m+1} & \ldots & \sum_{i=1}^{N} x_{i}^{2 m}
\end{array}\right]\left[\begin{array}{c}
a_{0} \\
a_{1} \\
\vdots \\
a_{m}
\end{array}\right]=\left[\begin{array}{c}
\sum_{i=1}^{N} y_{i} \\
\sum_{i=1}^{N} x_{i} y_{i} \\
\vdots \\
\sum_{i=1}^{N} x_{i}^{m} y_{i}
\end{array}\right] .
$$

\section{Damage index based on the changes in curvatures}

Structural damage causes structural stiffness to change, and the changes in stiffness will result in the changes in the curvature of the structure under load. Therefore, the changes in curvature of the structure between intact members and damaged ones can be used as damage index.

The ratio of the curvature of intact member to that of damaged one is

$$
R I D=\left|\rho_{0}\left(x_{i}\right) / \rho_{1}\left(x_{i}\right)\right| \text {. }
$$

Where $\rho_{0}\left(x_{i}\right)$ and $\rho_{1}\left(x_{i}\right)$ are the curvatures of intact and damaged member at $x_{i} . \rho(x)$ can be calculated using the following equation

$$
\rho(x)=\frac{\left|y^{\prime \prime}\right|}{\left(1+\left(y^{\prime}\right)^{2}\right)^{3 / 2}} .
$$

Where $y$ is the expression of the fitted curve.

Curvature change rate is defined as

$$
C C R=\left(\rho_{1}\left(x_{i}\right)-\rho_{0}\left(x_{i}\right)\right) / \rho_{0}\left(x_{i}\right) .
$$

\section{Numerical example}

Damage detection was conducted for a curved beam with quarter-arc shape, as show in Fig. 1. The radius, modulus of elasticity, unit weight and Poisson's ratio are $2.0 \mathrm{~m}, 36 \mathrm{GPa}, 26 \mathrm{kN} / \mathrm{m}^{3}$ and 0.33 , respectively. The beam is subjected to vertical uniform load, $1 \mathrm{kN} / \mathrm{m}$. 


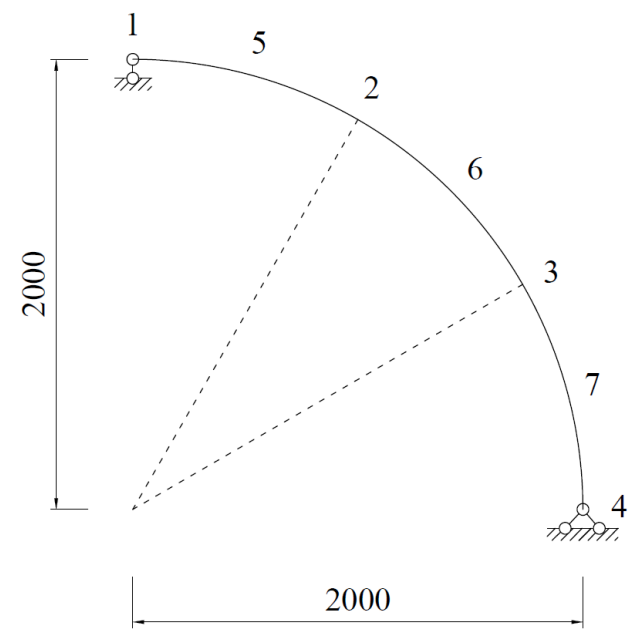

Fig. 1. Diagram of curved beam

The beam was divided into three segments, $P_{1} P_{2}, P_{2} P_{3}$ and $P_{3} P_{4}$. Each segment was fitted using quadratic polynomial, which requires three points for every segment. Segment $P_{1} P_{2}$ includes points 1, 5 and 2; Segment $P_{2} P_{3}$ includes points 2, 6 and 3; Segment $P_{3} P_{4}$ includes points 3, 7 and 4.

When the vertical uniform load is applied to the beam, the coordinates of all points can be calculated by finite element analysis. For example, the coordinates of points 1, 5 and 2 are listed in Table 1.

Table 1. The coordinates of points 1,5 and 2 of intact beam

\begin{tabular}{cccc}
\hline$i$ & 1 & 5 & 2 \\
\hline$x_{i}$ & 0 & 0.39194 & 0.75482 \\
\hline$y_{i}$ & 2 & 1.74112 & 1.42789 \\
\hline
\end{tabular}

Substituting data in Table 1 into Eq. (4), the equation of segment $P_{1} P_{2}$ can be obtained as follows

$$
y_{1}^{0}(x)=2-0.55528 x-0.26849 x^{2}
$$

Similarly, the equations of segments $P_{2} P_{3}$ and $P_{3} P_{4}$ also can be obtained

$$
\begin{aligned}
& y_{2}^{0}(x)=2.08740-0.80699 x-0.08843 x^{2}, \\
& y_{3}^{0}(x)=0.86440+0.81042 x-0.62131 x^{2} .
\end{aligned}
$$

When segment $P_{1} P_{2}$ is damaged with stiffness loss of $20 \%$, the coordinates of points 1,5 and 2 are listed in Table 2.

Table 2. The coordinates of points 1,5 and 2 of damaged beam

\begin{tabular}{cccc}
\hline$i$ & 1 & 5 & 2 \\
\hline$x_{i}$ & 0 & 0.36636 & 0.71395 \\
\hline$y_{i}$ & 2 & 1.72504 & 1.41357
\end{tabular}

According to the data in Table 2 , the equation of segment $P_{1} P_{2}$ of damaged beam was obtained as follows

$$
y_{1}^{1}(x)=2-0.67583 x-0.20388 x^{2}
$$

The equations of segments $P_{2} P_{3}$ and $P_{3} P_{4}$ have the following form

$$
\begin{aligned}
& y_{2}^{1}(x)=2.02580-0.80456 x-0.07419 x^{2}, \\
& y_{3}^{1}(x)=0.95499+0.63503 x-0.55626 x^{2}
\end{aligned}
$$


Substituting Eqs. (8), (9), (10), (11), (12) and (13) into Eqs. (5), (6) and (7), RID and CCR can be obtained. RID and $C C R$ are show in Fig. 2.
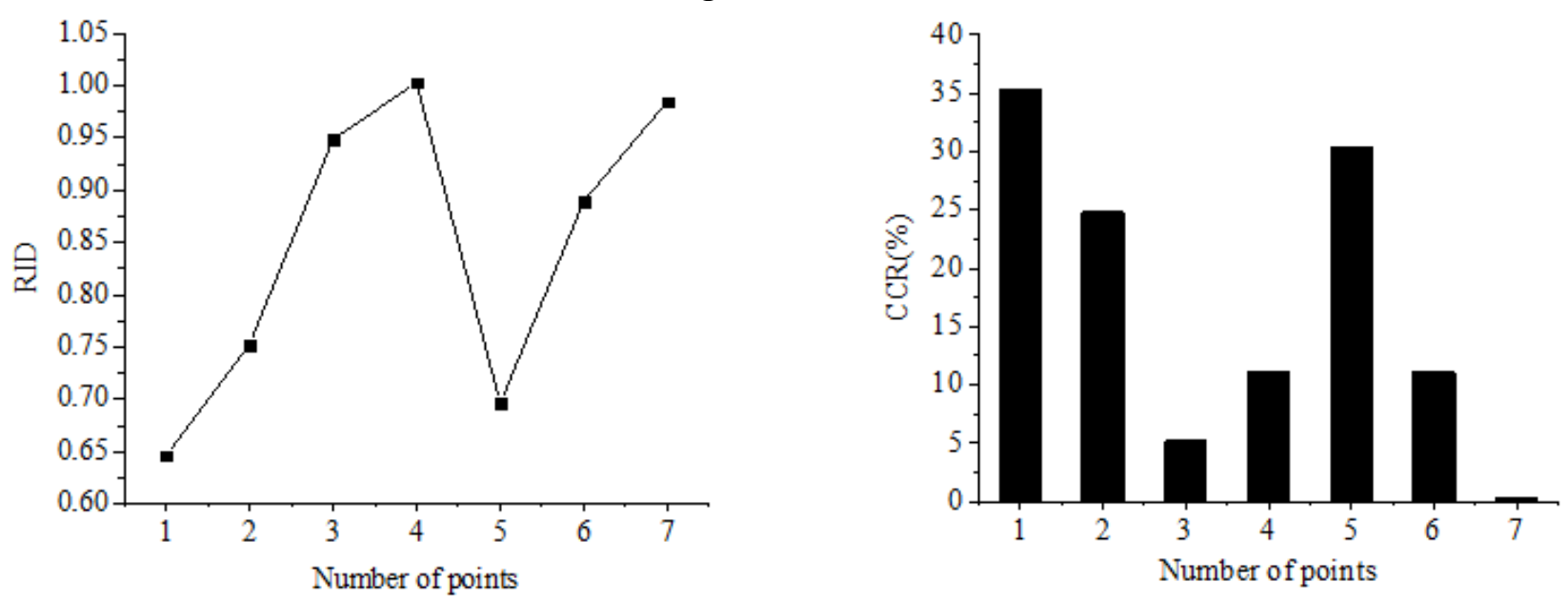

Fig. 2. RID and $C C R$ of all points

It can be found that the RID s of points 1,5 and 2 are obviously smaller than those of other points, and the $C C R$ of points 1,5 and 2 are obviously greater than those of other points. Therefore, the segment $P_{1} P_{2}$ can be identified as damaged one, which is consistent with actual damage location.

\section{Conclusion}

The stiffness of structural member decreases due to damage, which makes the curvature of the member change under load. Therefore, the changes in the curvatures can be treated as indices for damage identification, and a damage detection method was developed for curved structural member. The method was demonstrated by identifying the damage location of a curved beam. The results show that the changes in the curvatures are effective indices for damage location.

\section{Acknowledgement}

The authors acknowledge the financial support by the National Natural Science Foundation of China (Grant No. 51278214) and the Natural Science Foundation of Fujian Province, China (Grant No. 2017J01679).

\section{References}

[1] H. Ding, and X. H. Jiang, Optimization of disease classification method for road tunnel seepage, Technology of Highway and Transport, vol.15, pp. 89-94, 2017.

[2] J. B. Wu, D. L. Zhang, M. S. Wang, Current damage situation of railway operation tunnels and their inspection and evaluation, China Safety Science Journal. vol. 13, pp. 49-53, 2003.

[3] J. M. Ndambi, J. Vantomme, K. Harri, Damage assessment in reinforced concrete beams using eigenfrequencies and mode shape derivatives, Engineering Structures, vol. 24, pp. 501-515, 2002.

[4] J. T. Kim, Y. S. RYU, H. M. Cho, et al. Damage identification in beam-type structures: Frequency-based method vs mode-shape-based method. Engineering Structures, vol. 25, pp. 5767, 2003. 